\title{
High expression of microRNA-127 is involved in cell cycle arrest in MC-3 mucoepidermoid carcinoma cells
}

\author{
JI-AE SHIN* , CHENGMING LI* , EUN-SUN CHOI, SUNG-DAE CHO and NAM-PYO CHO \\ Department of Oral Pathology, School of Dentistry, Institute of Oral Bioscience, \\ Chonbuk National University, Jeonju, Republic of Korea
}

Received August 6, 2012; Accepted November 30, 2012

DOI: $10.3892 / \mathrm{mmr} .2012 .1222$

\begin{abstract}
RNAs (miRs) are small endogenous non-coding RNAs and are associated with the pathogenesis of a number of types of human cancer. However, miR-127-3p in mucoepidermoid carcinoma (MEC) has not been studied. The present study aimed to analyze the importance of miR-127-3p in MC-3 human MEC cells. Analyses of the growth inhibitory effect and the associated mechanism of miR-127-3p were performed using 3-(4,5-dimethylthiazol-20yl)-(3-carboxymethoxyphenyl)-2-(4-sulphophenyl)-2H-tetrazolium assay, flow cytometry, 4'-6-diamidino-2-phenylindole staining, anchorage-independent cell transformation assay and western blot analysis. Transfection of exogenous miR-127-3p into MC-3 cells inhibited cell viability and led to $G_{1} / S$ cell cycle arrest. In addition, miR-127-3p also decreased neoplastic cell transformation in TPA-induced JB6 mouse epidermal and MC-3 cells. In addition, miR-127-3p decreased specificity protein 1 (Sp1) expression and increased p21 and p27 expression which are $\mathrm{Sp1-dependent} \mathrm{cell} \mathrm{cycle-related} \mathrm{proteins.} \mathrm{However,}$ miR-127-3p did not induce apoptosis or affect expression levels of myeloid cell leukemia-1 or survivin. miR-127-3p induced $\mathrm{G}_{1} / \mathrm{S}$ cell cycle arrest and increased $\mathrm{p} 21$ and $\mathrm{p} 27$ expression via modulation of Sp1. Therefore, miR-127-3p may be a therapeutic target for human MEC.
\end{abstract}

\section{Introduction}

Salivary gland tumors are relatively uncommon lesions accounting for 3-6\% of all head and neck neoplasms (1) and the annual worldwide incidence is 0.4-13.5 new cases per 100,000 individuals (2-4). Mucoepidermoid carcinoma

Correspondence to: Dr Sung-Dae Cho or Dr Nam-Pyo Cho, Department of Oral Pathology, School of Dentistry and Institute of Oral Bioscience, Chonbuk National University, 664-14 Duckjindong 1ga, Duckjingu, Jeonju 561-756, Republic of Korea

E-mail: efiwdsc@chonbuk.ac.kr

E-mail:npcho@jbnu.ac.kr

*Contributed equally

Key words: miR-127-3p, mucoepidermoid carcinoma, cell cycle, Sp1, p21, p27
(MEC) is the most common malignant tumor of the salivary glands, representing 30-40\% of all salivary gland malignancies (5). As with the majority of salivary gland tumors, MEC demonstrates morphological diversity and variable biological behavior (6). Therefore, an understanding of carcinogenic mechanisms involved in MEC may offer new target candidates for prognostic and therapeutic strategies $(7,8)$.

microRNAs (miRs) are small endogenous non-coding RNAs that regulate the expression of their target genes at the post-transcriptional level (9). To date, $>1,420$ miRNAs have been identified in humans (miRBase v17). Each miRNA suppresses multiple mRNA targets (average, 200) and it is estimated that more than one-third of all human genes are regulated by miRNA molecules (10). Emerging evidence indicates that miRNAs may be associated with the pathogenesis of various types of human cancer (11). The frequent aberrant expression and functional implication of miRNAs in human cancer may be useful as important diagnostic and prognostic biomarkers, as well as potential therapeutic targets (12). However, the importance of miRNA-127-3p in MEC carcinogenesis has not been studied. In the present study, we investigated the growth inhibitory effect of miR-127-3p and the molecular mechanism of this RNA molecule in MC-3 human MEC cells.

\section{Materials and methods}

Reagents. FAM-labeled pre-miR negative control \#1 (miR-FAM) and hsa-miR-127 pre-miR miRNA precursor (miR-127-3p) were supplied by Ambion (Austin, TX, USA). Antibodies against Sp1, p21, p27 and actin were purchased from Santa Cruz Biotechnology, Inc. (Santa Cruz, CA, USA). PARP antibody was obtained from BD Pharmingen (San Jose, CA, USA). Antibodies against Mcl-1 and survivin were provided by Cell Signaling Technology, Inc. (Danvers, MA, USA).

Cell culture and chemical treatment. MC-3 human MEC cells were provided by Professor Wu Junzheng (Forth Military Medical University, Xi'an, China). Cells were cultured in DMEM supplemented with $10 \%$ fetal bovine serum (FBS) and antibiotics at $37^{\circ} \mathrm{C}$ in a $5 \% \mathrm{CO}_{2}$ incubator. JB6 mouse skin epidermal cells were obtained from the American Tissue Culture Collection (Manassas, VA, USA). Cells were cultured in MEM supplemented with $5 \% \mathrm{FBS}$ and antibiotics at $37^{\circ} \mathrm{C}$ in a $5 \% \mathrm{CO}_{2}$ incubator. Cells were transfected with miR-FAM 
A

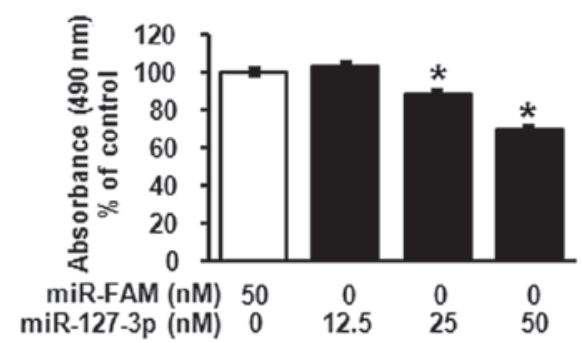

B

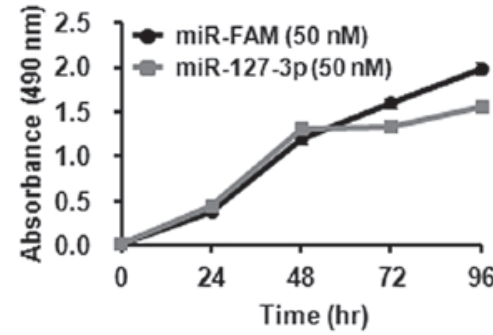

C
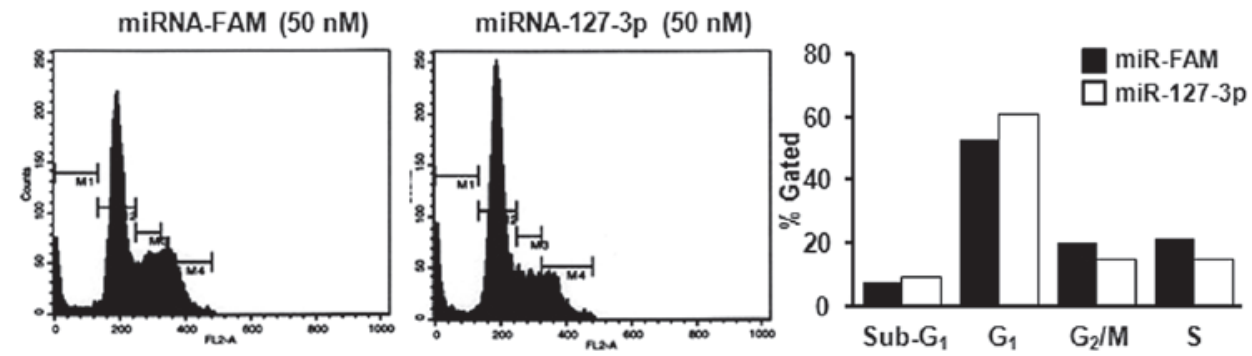

Figure 1. (A) MC-3 cells were transfected with miR-FAM or various concentrations $(12.5,25$ and $50 \mathrm{nM})$ of miR-127-3p for $96 \mathrm{~h}$. The growth inhibitory effect of miR-127-3p was determined using the MTS assay. Graphs present the results of triplicate experiments. ${ }^{*} \mathrm{P}<0.05$, vs. miR-FAM-transfected cells. (B) miR-FAM- or $50 \mathrm{nM}$ miR-127-3p-transfected MC-3 cells were analyzed using the MTS assay at various time-points (24, 48, 72 and $96 \mathrm{~h}$ ). (C) Cell cycle profiles of MC-3 cells transfected with miR-FAM or $50 \mathrm{nM}$ miR-127-3p were subjected to propidium iodide staining and flow cytometry analysis after $96 \mathrm{~h}$.

or miR-127-3p at various time points $(24,48,72$ or $96 \mathrm{~h})$ and concentrations $(12.5,25$ or $50 \mathrm{nM})$.

MTS assay. Effect of miR-127-3p on cell viability was measured using the CellTiter 96 Aqueous One Solution Cell Proliferation Assay kit (Promega, Madison, WI, USA). MC-3 cells were seeded in 96-well plates and then incubated for 24, 48,72 or $96 \mathrm{~h}$ with various concentrations of miR-127-3p. Absorbance was measured at 490 and $690 \mathrm{~nm}$ (background) using an ELISA microplate reader (Bio-Tek Instruments, Inc., Madison, WI, USA).

Flow cytometry analysis. Following transfection with miR-FAM or miR-127-3p for $96 \mathrm{~h}$, cells were harvested by trypsinization and fixed overnight in $70 \%$ ethanol at $-20^{\circ} \mathrm{C}$. The fixed cells were subsequently centrifuged, resuspended in PBS and stained with $0.02 \mathrm{mg} / \mathrm{ml}$ propidium iodide. The population of cells in each of the sub- $G_{1}, G_{1}, S$ and $G_{2} / M$ phases was then determined for $\sim 200,000$ cells. Cell cycle position was analyzed by FACScan cytometer.

Anchorage-independent cell transformation assay. The effect of miR-127-3p on cell transformation was determined in JB6 or MC-3 cells. TPA ( $20 \mathrm{ng} / \mathrm{ml})$-stimulated JB6 or MC-3 cells were transfected with miR-FAM or miR-127-3p. Cells were then incubated in $1 \mathrm{ml}$ of $0.3 \%$ basal medium Eagle's agar containing $10 \%$ FBS. Cultures were maintained at $37^{\circ} \mathrm{C}$ in a $5 \% \mathrm{CO}_{2}$ incubator for 10 days (JB6) or 20 days (MC-3), followed by analysis of colony numbers.

Western blot analysis. Whole cell lysates were extracted by lysis buffer and protein concentration of these lysates was quantified using the DC Protein Assay (Bio-Rad Laboratories, Hercules, CA, USA). Samples containing equal amounts of protein were separated by SDS-PAGE and then transferred to Immun-Blot PVDF membranes (Bio-Rad Laboratories). Membranes were blocked with 5\% skimmed milk in TBST at room temperature for $2 \mathrm{~h}$ and incubated overnight at $4^{\circ} \mathrm{C}$ with primary antibodies against PARP, Sp1, p21, p27, cyclin D1 or actin, followed by incubation with HRP-conjugated secondary antibodies. The antibody-bound proteins were detected using the ECL Western Blotting Luminol reagent (Santa Cruz Biotechnology, Inc.).

Statistical analysis. Data are presented as the mean \pm SD of triplicate samples from at least 3 independent experiments. Statistical significance was evaluated using a Student's t-test or one-way ANOVA. $\mathrm{P}<0.05$ was considered to indicate a statistically significant difference.

\section{Results}

Growth inhibitory effect of miR-127-3p is associated with $G_{I} / S$ arrest. The effect of miR-127-3p on MC-3 human MEC cells was investigated. Cells were transfected with miR-FAM or various concentrations $(12.5,25$ and $50 \mathrm{nM})$ of miR-127-3p for $96 \mathrm{~h}$ and cell viability was examined using the MTS assay. A high concentration of miR-127-3p was identified to significantly decrease cell viability in MC-3 cells $(\mathrm{P}<0.05)$, whereas $50 \mathrm{nM}$ of miR-FAM did not change cell viability (Fig. 1A). The time-dependent effect of miR-127-3p on cell viability was also examined. The results demonstrate that $50 \mathrm{nM} \mathrm{miR-127-3p}$ decreased cell viability in a time-dependent manner compared with miR-FAM-transfected cells (Fig. 1B). Following this, the growth inhibitory effect of miR-127-3p on cell cycle arrest was analyzed. Fig. 1C demonstrates that $50 \mathrm{nM}$ miR-127-3p caused an elevation in the $G_{1}$ cell population, indicating that it caused $\mathrm{G}_{\mathrm{l}} / \mathrm{S}$ arrest. These results suggest that the growth inhibitory effect of miR-127-3p is induced by $\mathrm{G}_{1} / \mathrm{S}$ cell cycle arrest.

miR-127-3p decreases anchorage-independent colony formation in vitro. To determine the significance of miR-127-3p in neoplastic cell transformation, JB6 cells with TPA were 


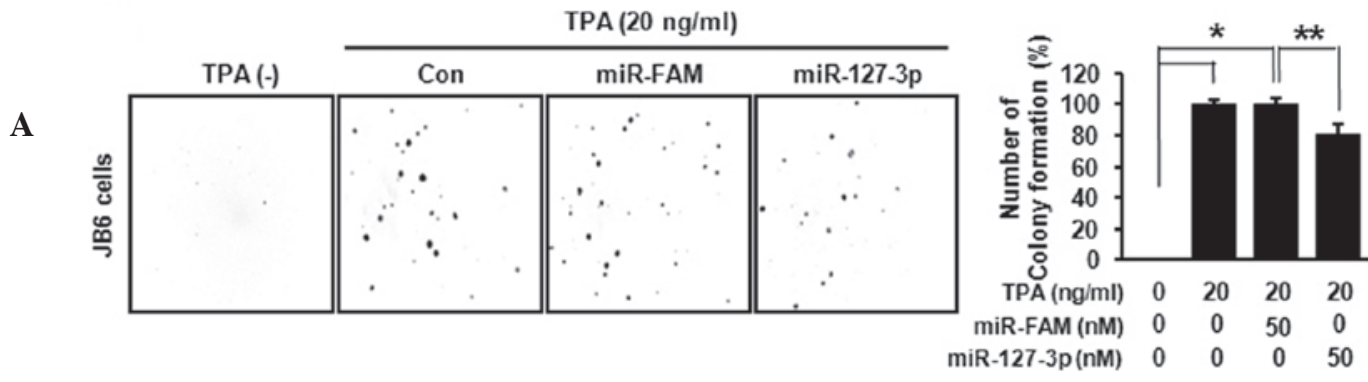

B

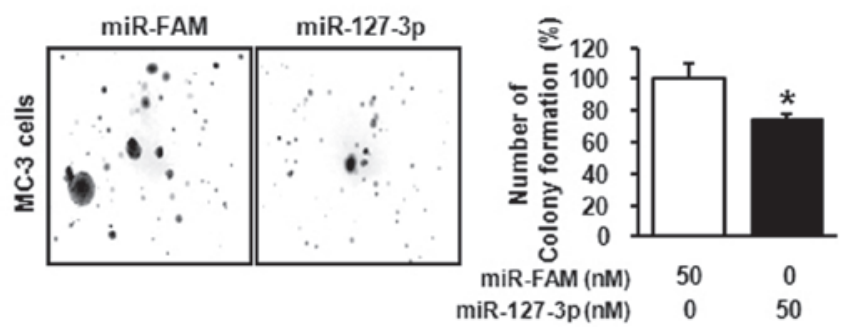

Figure 2. (A) Anchorage-independent cell transformation assay. JB6 mouse skin epidermal cells were stimulated by TPA (20 ng/ml), transfected with miR-FAM or miR-127-3p and cultured in $1 \mathrm{ml}$ of $0.3 \%$ basal medium Eagle's agar containing $10 \%$ FBS for 10 days. Cell colonies were counted. "P<0.05, vs. non-treated cells. ${ }^{* *} \mathrm{P}<0.05$, vs. miR-FAM-transfected cells. (B) MC-3 cells transfected with miR-FAM or miR-127-3p were cultured in $1 \mathrm{ml}$ of $0.3 \%$ BME agar containing $10 \%$ FBS for 20 days and cell colonies were counted. " $\mathrm{P}<0.05$, vs. miR-FAM-transfected cells. Graphs represent the mean of values obtained from the triplicate experiments.
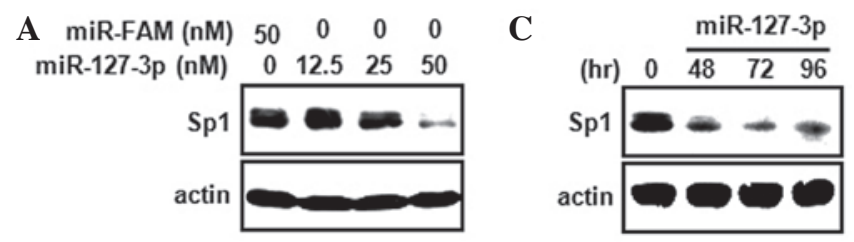

B miR-FAM (nM) $50 \quad 0 \quad 0 \quad 0$ $\begin{array}{lllll}\text { miR-127-3p (nM) } & 0 & 12.5 & 25 & 50\end{array}$
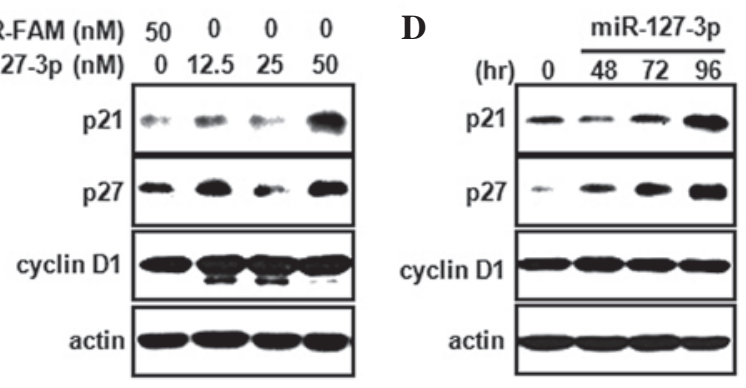

Figure 3. MC-3 cells were transfected with miR-FAM or various concentrations $(12.5,25$ and $50 \mathrm{nM})$ of $\mathrm{miR}-127-3 \mathrm{p}$ for $96 \mathrm{~h}$ and whole cell lysates were detected by western blot analysis using antibodies against (A) Sp1, (B) p27, p21 and cyclin D1. miR-FAM- or $50 \mathrm{nM}$ miR-127-3p-transfected MC-3 cells were harvested at various time points $(48,72$ and $96 \mathrm{~h})$ and protein levels of (C) Sp1, (D) p27, p21 and cyclin D1 were analyzed by western blot analysis. Equal loading was confirmed using actin protein.

analyzed using an anchorage-independent colony formation assay (20 ng/ml). miR-127-3p-transfected cells were identified to exhibit significantly fewer and smaller colonies than the miR-FAM-transfected cells (Fig. 2A). In addition, miR-127-3p-transfected MC-3 cells suppressed neoplastic cell transformation (Fig. 2B). These results indicate that miR-127-3p inhibits neoplastic cell transformation in vitro.

miR-127-3p downregulates Sp1 to induce p21 and p27 expression. Previous studies have demonstrated that several miRNAs regulate $\mathrm{Sp} 1$ expression, which is overexpressed in a number of types of tumor and cancer cell lines $(13,14)$. Therefore, we
A

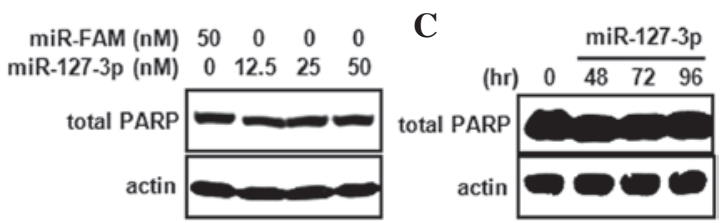

B

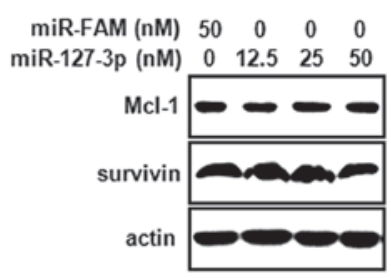

D

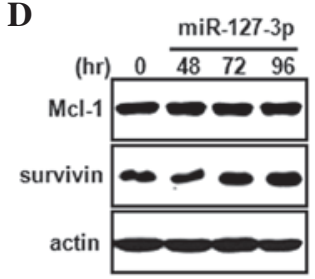

Figure 4. MC-3 cells were transfected with miR-FAM or various concentrations $(12.5,25$ and $50 \mathrm{nM})$ of miR-127-3p for $96 \mathrm{~h}$. Whole cell lysates were extracted and (A) PARP, (B) Mcl-1 and survivin levels were analyzed by western blot analysis. miR-FAM- or $50 \mathrm{nM}$ miR-127-3p-transfected cells were harvested at various time points (48, 72 and 96 h). (C) PARP, (D) Mcl-1 and survivin were detected by western blot analysis.

examined whether miR-127-3p regulates the expression of $\mathrm{Sp} 1$ protein in MC-3 cells. Fig. 3A and B demonstrate that miR-127-3p induced a concentration- and time-dependent decrease in Sp1 protein. In addition, the correlation between downregulation of $\mathrm{Sp} 1$ by miR-127-3p and $\mathrm{G}_{1} / \mathrm{S}$ arrest-related proteins, including p21, p27 and cyclin D1, was investigated. Fig. 3C and D demonstrate that a significant increase in protein levels of p21 and p27 was identified in cells treated with $50 \mathrm{nM}$ miR-127-3p compared with miR-FAM-transfected cells. This treatment did not affect cyclin D1. These results indicate that the downregulation of Sp1 by miR-127-3p increases p21 and p27 to cause $G_{1} / S$ arrest. Therefore, we hypothesize that $G_{1} / S$ arrest by miR-127-3p in MC-3 cells is associated with an increase of p21 and $\mathrm{p} 27$ expression via the modulation of Sp1 protein. 
Growth inhibitory effect of miR-127-3p is not associated with apoptosis. Next, the growth inhibitory activity of miR-127-3p was investigated to determine the correlation with apoptotic cell death. Fig. 4A and B demonstrate that miR-127-3p did not affect PARP protein levels. Mcl-1 and survivin, the downstream proteins of Sp1, were not altered in miR-127-3p-transfected cells (Fig. 4C and D). These results indicate that miR-127-3p does not inhibit cell growth by a mechanism associated with apoptotic cell death.

\section{Discussion}

miRNAs are endogenous short non-coding RNAs, which contain $\sim 22$ nucleotides. A previous study demonstrated that miRNAs regulate the expression of protein-coding genes by translational repression when complementary sequences are present in the 3'-untranslated regions of the target mRNAs or by directing mRNA degradation (15). miRNAs are expressed in a tissue-specific manner and are important for the regulation of a variety of cellular processes, including cell proliferation, apoptosis and differentiation (16-18). In particular, the deregulation of miRNAs is likely to contribute to numerous types of human diseases, including cancer (19). The expression levels of a number of miRNAs have been identified to be significantly decreased in specific types of cancer or cancer cell lines compared with normal tissues (20). Previous studies have identified that miRNAs are downregulated in various cancer cells (21-24). Overexpression of the lethal-7 family of miRNA regulates the expression of proto-oncogenes, including RAS and c-Myc, in cancer tissues (25-27). In addition, restoration of miR-122 in metastatic hepatocellular carcinoma cells was identified to significantly decrease migration, invasion and angiogenesis in vitro and in vivo (28) and overexpression of miR-330 induced apoptosis through E2F1-mediated suppression of Akt phosphorylation (29). Collectively, these previous studies demonstrate that miRNAs may have tumor suppressor activities in various types of cancer and cancer cell lines through the induction of cell death or apoptosis and regulation of oncogenes.

The aberrant regulation of miR-127, previously identified as miR-127-3p, has been observed in a number of human cancer types (30-32), which indicates tumor-suppressive roles of miRNAs in tumor development. Saito et al suggested that miR-127 is constitutively expressed in normal fibroblasts and normal tissues but silenced in cancer cells and downregulated in primary tumors in the prostate and bladder (31). A similar observation was reported by Tsai et al (33) identifying that the expression of tumor-suppressive miR-127-3p was significantly downregulated in gastric cancer. In addition, previous studies have revealed a mechanistic link between the downregulation of miR-127 and deregulated apoptosis during carcinogenesis $(34,35)$. These findings indicate the importance of miR-127 in carcinogenesis. In the present study, we identified that the exogenous insertion of miR-127-3p resulted in a marked elevation of the $\mathrm{G}_{1}$ cell population and decreased colony formation in MC-3 human MEC cells, suggesting that miR-127-3p expression may be associated with tumor suppression in MEC, consistent with the results of a previous study (33).

$\mathrm{Sp} 1$ protein is a critical transcription factor in tumor development, growth, angiogenesis and metastasis. Moreover, aberrant expression of Sp1 may contribute to cancer develop- ment and progression (36) and it is overexpressed in numerous types of human tumor and cancer cells $(37,38)$. A recent study indicated that miR-335 may function as a metastasis suppressor in gastric cancer by targeting Sp1 (14). Previously, our group also reported that $\mathrm{Sp} 1$ regulates proteins associated with cell survival $(38,39,40)$. Therefore, we hypothesized that miR-127-3p may regulate the Sp1 protein. Results demonstrate that miR-127-3p decreased Sp1 expression and induced downstream targets, p21 and p27, indicating that the $\mathrm{G}_{1} / \mathrm{S}$ arrest by miR-127-3p is associated with the modulation of Sp1. Previously, it was revealed that exogenous transfection of miR-29 resulted in loss of mitochondrial potential and release of cytochrome $\mathrm{c}$ into the cytoplasm in hepatocellular carcinoma cells, demonstrating that miR-29 induces apoptosis (41). miR-138 also induced apoptosis in head and neck squamous cell carcinoma cells (42). In addition, the downregulation of Bcl-2 by miR-15 and - 16 increased activation of the intrinsic apoptosis pathway in leukemic cells (43). These observations illustrate that miRs may affect apoptotic cell death in various types of cancer. Therefore, we investigated whether the exogenous transfection of miR-127-3p induces apoptotic cell death; however, no such effect was observed. Moreover, Mcl-1 and survivin, apoptosis-associated downstream targets of Sp1, were not affected by miR-127-3p. These results indicate that the growth inhibitory effect of miR-127-3p is markedly correlated with $\mathrm{G}_{1} / \mathrm{S}$ cell cycle arrest and not induction of apoptosis.

In summary, the importance of miR-127-3p in MC-3 MEC cells was investigated. We identified that miR-127-3p mediates antiproliferative activity through the suppression of cell cycle progression via $\mathrm{Sp} 1$ protein. Therefore, the present observations indicate that miR-127-3p may be a potential proliferation regulator targeting Sp1 protein against human MEC.

\section{Acknowledgements}

The present study was supported by the Basic Science Research Program through the National Research Foundation of Korea funded by the Ministry of Education, Science and Technology (2012001497, 2012002481 and 2012003731).

\section{References}

1. Ansari MH: Salivary gland tumors in an Iranian population: a retrospective study of 130 cases. J Oral Maxillofac Surg 65: 2187-2194, 2007.

2. Ma'aita JK, Al-Kaisi N, Al-Tamimi S and Wraikat A: Salivary gland tumors in Jordan: a retrospective study of 221 patients. Croat Med J 40: 539-542, 1999.

3. Jaber MA: Intraoral minor salivary gland tumors: a review of 75 cases in a Libyan population. Int J Oral Maxillofac Surg 35: 150-154, 2006.

4. Tian Z, Li L, Wang L, Hu Y and Li J: Salivary gland neoplasms in oral and maxillofacial regions: a 23 -year retrospective study of 6982 cases in an eastern Chinese population. Int J Oral Maxillofac Surg 39: 235-242, 2010.

5. McHugh CH, Roberts DB, El-Naggar AK, et al: Prognostic factors in mucoepidermoid carcinoma of the salivary glands. Cancer 118: 3928-3936, 2011.

6. Chiosea SI, Barnes EL, Lai SY, et al: Mucoepidermoid carcinoma of upper aerodigestive tract: clinicopathologic study of 78 cases with immunohistochemical analysis of Dicer expression. Virchows Arch 452: 629-635, 2008.

7. Mariano FV, da Silva SD, Chulan TC, de Almeida OP and Kowalski LP: Clinicopathological factors are predictors of distant metastasis from major salivary gland carcinomas. Int $\mathbf{J}$ Oral Maxillofac Surg 40: 504-509, 2011. 
8. Miyabe S, Okabe M, Nagatsuka H, et al: Prognostic significance of p27Kip1, Ki-67 and CRTC1-MAML2 fusion transcript in mucoepidermoid carcinoma: a molecular and clinicopathologic study of 101 cases. J Oral Maxillofac Surg 67: 1432-1441, 2009.

9. Bhaskaran M, Wang Y, Zhang H, et al: MicroRNA-127 modulates fetal lung development. Physiol Genomics 37 : 268-278, 2009.

10. Lewis BP, Burge CB and Bartel DP: Conserved seed pairing, often flanked by adenosines, indicates that thousands of human genes are microRNA targets. Cell 120: 15-20, 2005.

11. Pauley KM, Stewart CM, Gauna AE, et al: Altered miR-146a expression in Sjogren's syndrome and its functional role in innate immunity. Eur J Immunol 41: 2029-2039, 2011.

12. Hayashi T, Koyama N, Azuma Y and Kashimata M: Mesenchymal miR-21 regulates branching morphogenesis in murine submandibular gland in vitro. Dev Biol 352: 299-307, 2011

13. Wang F, Li Y, Zhou J, Xu J, Peng C, Ye F, Shen Y, Lu W, Wan X and Xie X: miR-375 is down-regulated in squamous cervical cancer and inhibits cell migration and invasion via targeting transcription factor SP1. Am J Pathol 179: 2580-2588, 2011.

14. Xu Y, Zhao F, Wang Z, Song Y, Luo Y, Zhang X, Jiang L, Sun Z, Miao Z and Xu H: MicroRNA-335 acts as a metastasis suppressor in gastric cancer by targeting Bcl-w and specificity protein 1. Oncogene 31: 1398-1407, 2012.

15. He L and Hannon GJ: MicroRNAs: small RNAs with a big role in gene regulation. Nat Rev Genet 5: 522-531, 2004.

16. Li B, Shi XB, Nori D, et al: Down-regulation of microRNA $106 \mathrm{~b}$ is involved in p21-mediated cell cycle arrest in response to radiation in prostate cancer cells. Prostate 71: 567-574, 2011.

17. Ambros V: The functions of animal microRNAs. Nature 431 : 350-355, 2004.

18. Sempere LF, Freemantle S, Pitha-Rowe I, Moss E, Dmitrovsky E and Ambros V: Expression profiling of mammalian microRNAs uncovers a subset of brain-expressed microRNAs with possible roles in murine and human neuronal differentiation. Genome Biol 5: R13, 2004.

19. Sassen S, Miska EA and Caldas C: MicroRNA: implications for cancer. Virchows Arch 452: 1-10, 2008

20. Lu J, Getz G, Miska EA, et al: MicroRNA expression profiles classify human cancers. Nature 435: 834-838, 2005.

21. Su H, Yang JR, Xu T, et al: MicroRNA-101, down-regulated in hepatocellular carcinoma, promotes apoptosis and suppresses tumorigenicity. Cancer Res 69: 1135-1142, 2009.

22. Song YX, Yue ZY, Wang ZN, et al: MicroRNA-148b is frequently down-regulated in gastric cancer and acts as a tumor suppresso by inhibiting cell proliferation. Mol Cancer 10: 1, 2011

23. Nadiminty N, Tummala R, Lou W, et al: MicroRNA let-7c is downregulated in prostate cancer and suppresses prostate cancer growth. PLoS One 7: e32832, 2012.

24. Nie J, Liu L, Zheng W, et al: microRNA-365, down-regulated in colon cancer, inhibits cell cycle progression and promotes apoptosis of colon cancer cells by probably targeting Cyclin D1 and Bcl-2. Carcinogenesis 33: 220-225, 2012

25. He XY, Chen JX, Zhang Z, Li CL, Peng QL and Peng HM: The let-7a microRNA protects from growth of lung carcinoma by suppression of k-Ras and c-Myc in nude mice. J Cancer Res Clin Oncol 136: 1023-1028, 2010.

26. Long XB, Sun GB, Hu S, et al: Let-7a microRNA functions as a potential tumor suppressor in human laryngeal cancer. Oncol Rep 22: 1189-1195, 2009

27. Johnson SM, Grosshans H, Shingara J, et al: RAS is regulated by the let-7 microRNA family. Cell 120: 635-647, 2005.
28. Tsai WC, Hsu PW, Lai TC, et al: MicroRNA-122, a tumor suppressor microRNA that regulates intrahepatic metastasis of hepatocellular carcinoma. Hepatology 49: 1571-1582, 2009.

29. Lee KH, Chen YL, Yeh SD, et al: MicroRNA-330 acts as tumor suppressor and induces apoptosis of prostate cancer cells through E2F1-mediated suppression of Akt phosphorylation. Oncogene 28: $3360-3370,2009$.

30. Yan LX, Huang XF, Shao Q, et al: MicroRNA miR-21 overexpression in human breast cancer is associated with advanced clinical stage, lymph node metastasis and patient poor prognosis. RNA 14: 2348-2360, 2008

31. Saito Y, Liang G, Egger G, et al: Specific activation of microRNA-127 with downregulation of the proto-oncogene BCL6 by chromatin-modifying drugs in human cancer cells. Cancer Cell 9: 435-443, 2006.

32. Zhang C, Wang C, Chen X, et al: Expression profile of microRNAs in serum: a fingerprint for esophageal squamous cell carcinoma. Clin Chem 56: 1871-1879, 2010.

33. Tsai KW, Wu CW, Hu LY, et al: Epigenetic regulation of miR-34b and miR-129 expression in gastric cancer. Int J Cancer 129 2600-2610, 2011.

34. Tryndyak VP, Ross SA, Beland FA and Pogribny IP: Down-regulation of the microRNAs miR-34a, miR-127 and miR-200b in rat liver during hepatocarcinogenesis induced by a methyl-deficient diet. Mol Carcinog 48: 479-487, 2009.

35. Duan Z, Choy E, Harmon D, et al: MicroRNA-199a-3p is downregulated in human osteosarcoma and regulates cell proliferation and migration. Mol Cancer Ther 10: 1337-1345, 2011.

36. Wei D, Wang L, He Y, Xiong HQ, Abbruzzese JL and Xie K: Celecoxib inhibits vascular endothelial growth factor expression in and reduces angiogenesis and metastasis of human pancreatic cancer via suppression of $\mathrm{Sp} 1$ transcription factor activity. Cancer Res 64: 2030-2038, 2004

37. Wang L, Wei D, Huang S, et al: Transcription factor Sp1 expression is a significant predictor of survival in human gastric cancer. Clin Cancer Res 9: 6371-6380, 2003.

38. Choi ES, Shim JH, Jung JY, et al: Apoptotic effect of tolfenamic acid in androgen receptor-independent prostate cancer cell and xenograft tumor through specificity protein 1 . Cancer Sci 102: 742-748, 2011.

39. Shim JH, Shin JA, Jung JY, et al: Chemopreventive effect of tolfenamic acid on KB human cervical cancer cells and tumor xenograft by downregulating specificity protein 1 . Eur J Cancer Prev 20: 102-111, 2011.

40. Shin JA, Shim JH, Jeon JG, et al: Apoptotic effect of Polygonum Cuspidatum in oral cancer cells through the regulation of specificity protein 1. Oral Dis 17: 162-170, 2011.

41. Xiong Y, Fang JH, Yun JP, et al: Effects of microRNA-29 on apoptosis, tumorigenicity and prognosis of hepatocellular carcinoma. Hepatology 51: 836-845, 2010.

42. Liu X, Jiang L, Wang A, Yu J, Shi F and Zhou X: MicroRNA-138 suppresses invasion and promotes apoptosis in head and neck squamous cell carcinoma cell lines. Cancer Lett 286: 217-222, 2009.

43. Cimmino A, Calin GA, Fabbri M, et al: miR-15 and miR-16 induce apoptosis by targeting BCL2. Proc Natl Acad Sci USA 102: 13944-13949, 2005. 